\title{
Subjective Symptoms and Serum Methadone Concentration: What Should Guide Dose Adjustments in Methadone Maintenance Treatment? A Naturalistic Cohort Study From Norway
}

Fatemeh Chalabianloo ( $\square$ fatemeh.chalabianloo@helse-bergen.no)

Haukeland University Hospital https://orcid.org/0000-0003-1242-1972

\section{Lars Thore Fadnes}

Haukeland University Hospital: Haukeland Universitetssjukehus

\section{Gudrun Høiseth}

Oslo University Hospital: Oslo Universitetssykehus

\section{Christian Ohldieck}

Haukeland University Hospital: Haukeland Universitetssjukehus

Jørn Henrik Vold

University of Bergen: Universitetet i Bergen

\section{Christer Aas}

University of Bergen: Universitetet i Bergen

\section{Else-Marie Løberg}

Haukeland University Hospital: Haukeland Universitetssjukehus

\section{Kjell Arne Johansson}

Haukeland University Hospital: Haukeland Universitetssjukehus

\section{Jørgen G. Bramness}

Norwegian Institute of Public Health Knowledge Centre for the Health Services: Folkehelseinstituttet Formidling og bruk av kunnskap

\section{Research}

Keywords: Methadone maintenance treatment, serum concentrations, subjective opioid withdrawal symptoms, adverse effects, substance use, opioid agonist treatment

Posted Date: January 13th, 2021

DOI: https://doi.org/10.21203/rs.3.rs-143001/v1 
License: (c) (i) This work is licensed under a Creative Commons Attribution 4.0 International License. Read Full License 


\section{Abstract}

\section{Background}

There is little evidence-based guidance on how to optimize methadone dosage among patients with opioid addiction undergoing methadone maintenance treatment (MMT). This study aims to investigate whether self-perceived opioid withdrawal symptoms, adverse effects and self-reported substance use in patients on MMT are related to serum methadone concentration and what role these could play in clinical decisions on dose adjustments.

\section{Methods}

In this naturalistic cohort study clinical and laboratory measurements from 83 patients undergoing MMT in outpatient clinics in Bergen, Norway during May 2017-January 2020 were included. Information on age, gender, methadone daily doses and serum concentrations, subjective opioid withdrawal symptoms (SOWS), self-reported adverse effects and substance use were obtained. Linear mixed modelling was used for analyzing the data.

Results

Mean age was 45 years and $34 \%$ were women. $55 \%$ reported subjective opioid withdrawal symptoms, and all had experienced at least one subjective adverse effect. Self-reported substance use was recorded in $88 \%$ of the interviews. Total SOWS score $(P<0.001)$, and the specific subjective withdrawal symptoms of anxiety $(P=0.004)$, bone- and muscle ache $(P=0.003)$, restlessness $(P=0.017)$ and shaking $(P=0.046)$ out of the 16 symptoms in standard SOWS questionnaire, as well as the use of heroin $(P=0.015)$ and alcohol $(P=0.011)$ were associated with lower methadone concentrations, whereas cannabis use was associated with higher methadone concentrations $(P=0.049)$.

\section{Conclusions}

More subjective opioid withdrawal symptoms and more self-reported use of heroin and alcohol were associated with lower serum methadone concentrations. More use of cannabis was related to higher serum methadone concentrations. These findings suggest that patient's self-perceived symptoms and current clinical condition can be applied as the main guide in methadone dose adjustments. In some aberrant cases, measurement of serum concentration together with other individual assessments may be considered to support the clinical decision.

\section{Background}

Methadone maintenance treatment (MMT) is an evidence-based medical intervention that reduces illicit opioid use and risk of overdose [1, 2] and mortality [3, 4] among opioid dependent individuals. Understanding factors that may influence treatment satisfaction and continuity, and accordingly prevent relapse into illicit opioid use with the following risks of overdose and death is crucial. Such factors may 
be opioid withdrawal symptoms and adverse effects related to inappropriate methadone dosages. This emphasizes the importance of balancing an efficacious dose to achieve the desired therapeutic effect, against either a too low dose, leading to withdrawal symptoms and relapse to illicit opioid use or a too high dose, causing adverse effects and toxicity.

Individualized dose optimization using doses between 60-120 mg daily for most of the patients appears to be related to increased retention in treatment and reduced illicit opioid use as the most common measures of efficacy of MMT [5]. However inter-individual variations in methadone dose requirements should be kept in mind [6-8]. Furthermore, some researchers have shown that other factors than dose, such as patients' expectations and preferences of medication as well as the total physical and mental health condition or improvement in psychosocial function may influence treatment satisfaction $[9,10]$. These findings add to the complexity of the issue challenging clinicians in how to cope with a suboptimal treatment outcome; should the dose be adjusted or should other problems rather be addressed.

The optimal methadone maintenance dose may be predicted by various factors based on continuous clinical evaluations [11-13]. Although previous findings suggest an association between dose and clinical symptoms, the relationships between serum methadone concentration and treatment effects are still not fully understood [14]. Due to a large inter-individual variation in methadone pharmacokinetics, the individual serum concentration after a given dose will vary substantially [12,14-16]. The optimal dose at steady state thus is hard to predict [12]. Accordingly, a clinical-oriented approach rather than serum levels has been suggested in order to optimize methadone daily dosage for the individual patient $[11,12,17]$.

Serum concentration, however, is probably a better indicator of the drug exposure at effect site compared to dose. Some researchers $[18,19]$ have suggested a direct association between serum methadone concentration and retention in treatment. A few clinical studies $[20,21]$ have shown that when the serum concentration is too low to inhibit the objective withdrawal symptoms, the patients relapse to substance use and drop out of MMT. Other studies have investigated its relationship to craving $[11,22,23]$ indicating that higher serum concentrations of methadone are more likely to reduce craving to opioids. Finally, a rapid decline, but not the level of trough concentration, has shown to be related to clinically important responses, notably the objective withdrawal symptoms [24]. These sparse findings indicate an association between methadone serum concentration and clinical effects. However, there is limited knowledge about the specific self-perceived symptoms reflecting a low or high serum concentration of methadone that clinicians need to be aware of as a guide for proper dose adjustments in clinical practice. The present study did not aim to assess an association between objective treatment effects and certain serum concentrations, but rather used the supplementary tool of therapeutic drug monitoring (TDM) in better understanding the importance of clinical evaluations based on self-perceived symptoms in MMT.

We believe that identifying the specific subjective symptoms that are related to serum methadone concentration could help clinicians find the individualized methadone doses based on patient-reported symptoms to could improve treatment outcomes. The present study aims to investigate whether selfperceived opioid withdrawal symptoms, adverse effects and substance use in patients on MMT are 
related to serum methadone concentration and what role these could play in clinical decisions on dose adjustments.

\section{Methods}

\section{Design, setting and data sources}

Participants for this naturalistic cohort study were recruited from the Department of Addiction Medicine, Haukeland University Hospital in Bergen, Norway. The department is responsible for the treatment and follow-up of more than 1000 patients with opioid dependence receiving opioid agonist treatment (OAT), of which almost $40 \%$ receive MMT while the rest are mainly substituted with buprenorphine-based treatment. All pharmacological interventions are integrated with psychosocial treatment and are provided in multidisciplinary outpatient clinics. The patients are followed-up by direct observed treatment (DOT) and consultations with different frequencies of take-home doses based on the individual treatment courses. All the clinical measurements and laboratory data are recorded prospectively in the hospital journal system as well as in a recently established health registry database for patients undergoing OAT in the city of Bergen. In addition to individual health data, the database includes relevant information based on clinical surveys and research records.

In the present study, we included information on age, gender, methadone daily doses, serum methadone concentrations (as the independent variable), subjective opioid withdrawal symptoms (as the primary outcome), some common subjective opioid adverse effects and self-reported illicit drug use (as the secondary outcomes). We also included information about time since last dose intake, time of blood sampling, time when the symptoms were recorded, numbers of days with DOT per week and duration of OAT.

\section{Participants}

Among almost 400 patients on MMT in our department, 199 patients had consented to participate in the study and completed the primary surveys through in-person clinical interviews by a research nurse during May 2017 - January 2020. To enable comparable measurements, we included only the data where the time difference between clinical measurements and laboratory data collection was shorter than 14 days. The mean time difference between blood sampling and recording of the different outcome symptoms for all the 199 patients was 106 (95\% confidence interval: 82-131) days. Eighty-nine of the records were within a timeframe of less than 14 days with a mean difference of $3(2-3)$ days. Therefore, we included the 89 measurements of the clinical data obtained from 83 participants in this study meaning that 6 out of 83 participants had two sett with clinical records attributed to two different serum concentration measurements, whereas the remaining had only one record of each during the study period.

\section{Serum methadone concentrations}

Blood samples were drown from the participants at the OAT clinics according to the study protocol with a mean time of $21(19-22)$ hours since the last dose intake, and centrifuged before transporting to the 
laboratory. Analysis of methadone in all the collected serum samples was performed by the same method using the same instruments. A validated and certified high-pressure liquid chromatographytandem mass spectrometry (HPLC-MS-MS) method was applied, developed at the Department of Medical Biochemistry and Clinical Pharmacology, Haukeland University Hospital, Bergen. In brief, the samples were extracted with ethyl acetate: heptane (4:1), before re-extraction and concentration in aqueous formic acid as the mobile phase. The components were separated on a short HPLC column (Phenomenex Kinetex XB-C18, 00B-4496-AN) by reverse phase chromatography. The column contained $2.6 \mu \mathrm{m}$ of silica particles with $\mathrm{C} 18$ (octadecyl) as the bound phase. MS-MS analysis was performed with electrospray ionization (ESI) in positive ion mode (Agilent Technologies 6410AA triple quadrupole LC-M-MS, CA, USA). The limit of quantification was $20 \mathrm{nmol} / \mathrm{L}$ and the method was linear at least to $4000 \mathrm{nmol} / \mathrm{L}$. Recoveries were $100 \%$ and $91 \%$ and inter-day coefficients of variation were $2.7 \%$ and $5.1 \%$ at low and high concentrations, respectively. During the development phase of the method as well as in routine use, methadone concentrations were measured in $\mathrm{nmol} / \mathrm{L}$. The conversion factor from $\mathrm{nmol} / \mathrm{L}$ to $\mathrm{ng} / \mathrm{mL}$ for methadone is 0.310 .

\section{Assessments of subjective symptoms}

As a part of the clinical assessments and based on the study protocol, the participants were initially asked if they experienced opioid withdrawal symptoms or not. Those who confirmed having withdrawal symptoms were interviewed by a research nurse using the validated standard questionnaire; Subjective Opioid Withdrawal Scale (SOWS) [25] covering 16 self-perceived symptoms including anxiety, yawning, perspiring, tearing, nose run, goose bumps, shaking, hot flushes, cold flushes, bone- and muscle ache, restlessness, nausea, vomiting, muscle twitches, stomach cramps and feeling like using, rated on a fivelevel scale from 0 (not at all) to 4 (extremely); total score: 0-64 (1-10= mild, 11-20 = moderate and 21$30=$ severe withdrawal).

In addition, all the participants were asked six questions on some of the most common subjective adverse effects attributed to MMT including euphoria, perspiring, nausea, concentration difficulties, feeling "brain fog" and reduced sexual desire, which were rated in the same way as done for the withdrawal symptoms. The selection of the adverse effects was based on our own clinical experiences, previously published peer collected data on this population [10] and the most common reported side effects for methadone [26, 27] and opioids in general [28]. Perspiring and nausea were defined as adverse effects or withdrawal symptoms based on how each participant had perceived these. There was also one open ended question on other possible symptoms when these were self-perceived to be related to MMT.

All clinical interviews and measurements of methadone serum concentration were performed before the daily doses of methadone were given, and no changes in dosages were made during the last week.

\section{Substance use}

Self-reported use of illicit drugs including heroin or other opioids, amphetamines (amphetamine and/or methamphetamine), benzodiazepines, cannabis, and also alcohol, as well as frequencies of use (categorized as; no use or frequent use i.e. several times a month, weekly or daily use) during the last 
month were recorded for the participants. Urine screening test was not used as the study focused on selfreports.

\section{Statistical analyses}

Basic descriptive statistical analysis of data was performed using Stata/SE 16.0 (StataCorp, TX, USA). Data were presented as means with $95 \%$ confidence interval for continuous variables. Comparisons of study variables in participants with and without reported subjective opioid withdrawal symptoms were performed using ANOVA (Mann-Whitney) test for continuous and chi-square test for categorical variables. The exact $p$-values were reported and values $<0.05$ were considered statistically significant. To avoid type-II statistical errors by overlooking some important variables due to naturalistic design of the study, all the variables with a $\mathrm{p}$-value $<0.10$ were included in the adjusted regression analyses.

Due to repeated measures for some participants, a linear mixed model (LMM) method was applied to investigate the possible associations between serum methadone concentrations and reported subjective symptoms. We included in the main analyses all the 16 SOWS items and the 6 subjective adverse effects, as well as self-reported substance use during the last month as dependent variables. The open question addressing any other reported undesirable symptoms was excluded from the statistical analyses due to few applicable data. All these variables were included one by one in the unadjusted statistical analysis when the time difference between blood sampling and record of the symptoms was less than 14 days. In total, 89 measurements obtained from 83 participants were included in the analyses. Then, adjusted LMM analyses for the specific variables having statistically significant association with serum methadone concentration were undertaken. Other variables were also included in adjusted LMM when the significance level was $<0.10$. The results obtained in the main analyses were adjusted for age, gender and absolute time difference between blood sampling and recording of the symptoms, but not for the time between last dose intake and sampling as this was within the acceptable range for all the measures.

\section{Results}

The mean age was $45(43-47)$ ranging from $26-62$ years with $34 \%$ being women. The mean daily dose of methadone was 98 (93-103) ranging from 20-170 mg and mean serum concentration was 388 (349-428) ranging from $123-984 \mathrm{ng} / \mathrm{mL}$ giving a mean concentration-to-dose ratio (CDR) of 13 (12-15) $(\mathrm{ng} / \mathrm{mL}) /(\mathrm{mg} / \mathrm{d})$.

More than half (55\%) reported mild to moderate subjective opioid withdrawal symptoms at the time of interviews with a mean total SOWS score of $9(6-11)$ having restlessness $(42 \%)$, hot flushes $(37 \%)$, cold flushes (36\%), anxiety (36\%), perspiring (35\%), nose run (33\%) and bone- and muscle ache (32\%) as the most frequently reported symptoms.

All of them (100\%) had experienced one or more subjective adverse effects with methadone treatment, most frequently reported as concentration difficulties (72\%), feeling "brain fog" (70\%), reduced sexual desire (63\%), perspiring (57\%) and nausea (37\%). 
Frequent substance use, more specifically cannabis (66\%), benzodiazepines (62\%), alcohol (46\%), amphetamines (31\%), heroin (12\%) and other opioids (6\%), was self-reported in $88 \%$ of the records during the last month prior to data collection.

Table 1 shows comparisons between participants who reported and those who did not reported to have subjective opioid withdrawal symptoms as the main outcome of the study. Serum concentration-to-dose ratios were higher among those who had reported subjective opioid withdrawals $(p=0.039)$. The daily doses of methadone did not significantly differ between the two groups. Those who reported withdrawal symptoms had also more frequently received DOT compared to the other group $(p=0.026)$. There were no differences between the groups with regard to age, gender or self-reported use of illicit drugs and alcohol. 
Table 1

Comparisons of clinical data in patients with and without reported opioid withdrawal symptoms $(n=89$ measurements from 83 participants)

\begin{tabular}{|c|c|c|c|c|c|}
\hline & & & $\begin{array}{l}\text { Opioid with } \\
\text { symptoms } \\
\text { Reported N }\end{array}$ & $\begin{array}{l}\text { awal } \\
\text { reported }\end{array}$ & \\
\hline & & & $N=49$ & $N=40$ & $p$-value $e^{a}$ \\
\hline Gender & female & n (\%) & $17(57)$ & $13(43)$ & 0.828 \\
\hline Age & years & $\begin{array}{l}\text { Mean }(95 \% \\
\left.\mathrm{Cl}^{b}\right)\end{array}$ & $\begin{array}{l}45(43- \\
48)\end{array}$ & $\begin{array}{l}45(42- \\
48)\end{array}$ & 0.753 \\
\hline Methadone dose & mg/day & $\begin{array}{l}\text { Mean }(95 \% \\
\mathrm{Cl})\end{array}$ & $\begin{array}{l}102(95- \\
109)\end{array}$ & $\begin{array}{l}93(85- \\
100)\end{array}$ & 0.079 \\
\hline $\begin{array}{l}\text { Methadone serum } \\
\text { concentration }\end{array}$ & $\mathrm{ng} / \mathrm{mL}$ & $\begin{array}{l}\text { Mean }(95 \% \\
\text { Cl) }\end{array}$ & $\begin{array}{l}364(316- \\
412)\end{array}$ & $\begin{array}{l}418(352- \\
485)\end{array}$ & 0.145 \\
\hline $\begin{array}{l}\text { Concentration-to-dose- } \\
\text { ratio }\end{array}$ & $(\mathrm{ng} / \mathrm{mL}) /(\mathrm{mg} / \mathrm{d})$ & $\begin{array}{l}\text { Mean }(95 \% \\
\mathrm{Cl})\end{array}$ & $\begin{array}{l}12(10- \\
14)\end{array}$ & $\begin{array}{l}15(13- \\
17)\end{array}$ & 0.039 \\
\hline Time since last dose & hours & $\begin{array}{l}\text { Mean }(95 \% \\
\mathrm{Cl})\end{array}$ & $\begin{array}{l}22(21- \\
24)\end{array}$ & $\begin{array}{l}19(16- \\
22)\end{array}$ & 0.199 \\
\hline Duration of OAT ${ }^{C}$ & years & $\begin{array}{l}\text { Mean }(95 \% \\
\mathrm{Cl})\end{array}$ & $9(7-10)$ & $10(8-11)$ & 0.602 \\
\hline Direct observed treatment & day/week & $\begin{array}{l}\text { Mean }(95 \% \\
\text { Cl) }\end{array}$ & $4(4-6)$ & $4(3-4)$ & 0.026 \\
\hline $\begin{array}{l}\text { Self-reported substance } \\
\text { use }^{d}\end{array}$ & & n (\%) & $45(92)$ & $33(83)$ & 0.187 \\
\hline Heroin & & $\mathrm{n}(\%)$ & $6(12)$ & $5(13)$ & 0.971 \\
\hline Other opioids & & n (\%) & $3(6)$ & $2(5)$ & 0.819 \\
\hline Benzodiazepines & & n (\%) & $33(64)$ & $22(55)$ & 0.233 \\
\hline Cannabis & & n (\%) & $32(65)$ & $27(67)$ & 0.828 \\
\hline Amphetamines & & n (\%) & $16(33)$ & $12(30)$ & 0.789 \\
\hline $\begin{array}{l}\text { a Significance was tested } \\
\text { categorical variables }\end{array}$ & g Mann-Whitney & est for con & lous and ch & uare test fol & \\
\hline${ }^{b}$ Confidence interval & & & & & \\
\hline${ }^{\mathrm{C}}$ Opioid agonist treatment & & & & & \\
\hline $\begin{array}{l}\text { d Frequent use (from daily } \\
\text { month }\end{array}$ & several times a mc & th) of illicit d & ugs and/or a & hol during th & last \\
\hline
\end{tabular}




\begin{tabular}{|c|c|c|c|}
\hline \multicolumn{4}{|c|}{$\begin{array}{ll} & \text { Opioid withdrawal } \\
\text { symptoms } \\
\text { Reported Not reported }\end{array}$} \\
\hline Alcohol & $24(49)$ & $17(42)$ & 0.542 \\
\hline \multicolumn{4}{|c|}{$\begin{array}{l}\text { a Significance was tested using Mann-Whitney U-test for continuous and chi-square test for } \\
\text { categorical variables }\end{array}$} \\
\hline \multicolumn{4}{|l|}{${ }^{b}$ Confidence interval } \\
\hline \multicolumn{4}{|l|}{${ }^{\mathrm{c}}$ Opioid agonist treatment } \\
\hline $\begin{array}{l}{ }^{d} \text { Frequent use (from daily to several times a month) of } \\
\text { month }\end{array}$ & Igs and/c & hol duri & last \\
\hline
\end{tabular}

The relationship between recorded total SOWS scores and the measured serum methadone concentrations is shown in Fig. 1, illustrating a weak correlation with wider confidence intervals at more lower and higher concentrations. When analyzing in the unadjusted LMM (Table 2), we found statistically significant inverse associations, although weak correlations, between serum methadone concentrations and total SOWS score $(p=0.011)$, but also for the specific symptoms of anxiety $(p=0.009)$, bone- and muscle ache $(p=0.007)$ and restlessness $(p=0.021)$ out of the 16 subjective opioid withdrawal symptoms based on SOWS questionnaire, and also use of heroin $(p=0.028)$ and alcohol $(p=0.008)$. None of the adverse medication effects were related to serum methadone concentrations, the exception being a significant positive association with nausea reported as an adverse effect of the treatment $(p=$ 0.040). 
Table 2

The unadjusted associations between serum methadone concentrations (measured in $\mathrm{ng} / \mathrm{mL}$ ) and the study variables. All the primary and secondary outcome variables were inserted one by one in linear mixed model ( $n=89$ measurements from 83 participants).

\begin{tabular}{|llll|}
\hline & Coefficient & $95 \% \mathbf{C l}^{\mathbf{a}}$ & $p$-value \\
\hline Total SOWS ${ }^{\mathrm{b}}$ score & -3.9 & $-7,-9.1$ & 0.011 \\
\hline Anxiety & -4.3 & $-7.6,-1.1$ & 0.009 \\
\hline Yawning & -0.5 & $-3.5,2.5$ & 0.739 \\
\hline Perspiring & -0.4 & $-4.1,3.3$ & 0.821 \\
\hline Tearing & -1.5 & $-4.1,1.2$ & 0.283 \\
\hline Nose run & -1.7 & $-4.9,1.5$ & 0.305 \\
\hline Goose bumps & -2.1 & $-5.5,1.4$ & 0.244 \\
\hline Shaking & -2.7 & $-5.7,0.3$ & 0.081 \\
\hline Hot flushes & -2.6 & $-6.2,8.9$ & 0.143 \\
\hline Cold flushes & -3.2 & $-7.1,0.8$ & 0.115 \\
\hline Bone- and muscle ache & -4.9 & $-8.4,-1.4$ & 0.007 \\
\hline Restlessness & -5.2 & $-9.6,-0.8$ & 0.021 \\
\hline Nausea & -1.5 & $-4.2,1.2$ & 0.272 \\
\hline Vomiting* & - & - & - \\
\hline Muscle twitches & -0.9 & $-4.2,2.4$ & 0.590 \\
\hline Stomach cramps & -1.5 & $-4.2,1.1$ & 0.263 \\
\hline Feel like using* & - & - & - \\
\hline Subjective adverse effects* & - & $-3,5.9$ & - \\
\hline Euphoria* & - & -526 \\
\hline Perspiring (as adverse effect) & -4 & - \\
\hline
\end{tabular}

a Confidence interval

${ }^{b}$ Subjective opioid withdrawal symptoms

${ }^{\mathrm{c}}$ Frequent use (from daily to several times a month) of illicit drugs and/or alcohol during the last month

*The linear mixed model system was not able to analyze these variables due to few data 


\begin{tabular}{|llll|}
\hline & Coefficient & $95 \%$ Cl $^{\mathrm{a}}$ & p-value \\
\hline Nausea (as adverse effect) & 3.1 & $0.1,6.1$ & 0.040 \\
\hline Concentration difficulties & -0.7 & $-4.7,3.3$ & 0.731 \\
\hline Feeling "brain fog" & 1.5 & $-2.2,5.2$ & 0.427 \\
\hline Decreased sexual desire & 2.2 & $-2.4,6.8$ & 0.351 \\
\hline Self-reported substance use c & 0.2 & $-0.9,1.3$ & 0.754 \\
\hline Heroin & -2 & $-3.8,-0.2$ & 0.028 \\
\hline Other opioids* & - & - & - \\
\hline Benzodiazepines & 0.6 & $-3.7,4.9$ & 0.772 \\
\hline Cannabis & 4.7 & $-0.5,9.9$ & 0.077 \\
\hline Amphetamines & -0 & $-3,2.9$ & 0.984 \\
\hline Alcohol & -4.5 & $-7.8,-1.2$ & 0.008 \\
\hline a Confidence interval & & & \\
\hline b Subjective opioid withdrawal symptoms & & & \\
\hline c Frequent use (from daily to several times a month) of illicit drugs and/or alcohol during the last \\
\hline month
\end{tabular}

When adjusting for age, gender and the absolute time difference between blood sampling and record of the symptoms in the LMM analyses (Table 3 ), the associations between serum methadone concentrations and total SOWS score, the specific withdrawal symptoms of anxiety, bone- and muscle ache, and restlessness, as well as the use of heroin and alcohol, still remained highly significant. However, the significance level fell down in the case of nausea reported as an adverse effect $(p=0.057)$. Obtaining the borderline $p$-values of $<0.10$ by analyzing the withdrawal symptom of shaking as well as use of cannabis in the unadjusted LMM, we also added these variables in the adjusted model and found slight significant associations with serum methadone concentrations $(p=0.046$ and $p=0.049$, respectively). 
Table 3

The adjusted* associations between serum methadone concentrations and the selected study variables

in linear mixed model based on obtained significance level in the unadjusted analysis $(n=89$ measurements from 83 participants)

\begin{tabular}{|llll|}
\hline & Coefficient & $95 \% \mathrm{Cl}^{\text {a }}$ & $p$-value \\
\hline Total SOWS ${ }^{\text {b Score }}$ & -4.3 & $-5.6,-2.9$ & $<0.001$ \\
\hline Anxiety & -0.5 & $-0.8,-0.2$ & 0.004 \\
\hline Bone- and muscle ache & -0.5 & $-0.9,-0.2$ & 0.003 \\
\hline Restlessness & -0.5 & $-9.7,-0.9$ & 0.017 \\
\hline Shaking & -0.3 & $-0.6,-0$ & 0.046 \\
\hline Nausea (as adverse effect) & 0.3 & $-0.1,0.6$ & 0.057 \\
\hline Heroin use & -0.2 & $-0.4,-0$ & 0.015 \\
\hline Alcohol use & -0.4 & $-0.7,-0.1$ & 0.011 \\
\hline Cannabis use & 0.5 & $0,10.4$ & 0.049 \\
\hline $\begin{array}{l}\text { *Adjusted for age, gender and absolute time difference between blood sampling and record of the } \\
\text { symptoms }\end{array}$ & & & \\
\hline a Confidence interval & & & \\
\hline b Subjective opioid withdrawal symptoms & & & \\
\hline a Subjective opioid withdrawal symptoms & & \\
\hline
\end{tabular}

\section{Discussion}

In this naturalistic cohort study, we used clinical and laboratory data from patients in MMT to investigate possible associations between self-perceived symptoms and serum methadone concentration. Restlessness, hot flushes, cold flushes, anxiety, nose run and bone- and muscle ache were the most frequently reported mild to moderate opioid withdrawal symptoms. There were weak inverse correlations but statistically significant associations between serum methadone concentration and such symptoms, represented by the total score of SOWS as well as the specific withdrawal symptoms of anxiety, boneand muscle ache, restlessness and shaking. Moreover, those with lower serum methadone concentrations more often had reported to use heroin and alcohol. All participants had experienced at least one subjective adverse effect related to methadone treatment, most frequently concentration difficulties, feeling "brain fog", reduced sexual desire, perspiring and nausea. There were, however, no significant relationships between serum methadone concentration and any of these subjective adverse effects in our analyses, except for a trend for more nausea among those having higher serum methadone 
concentrations. Also more cannabis use was reported among those with higher concentrations of methadone.

Previous research had found an inverse relationship between serum methadone concentration and general objective withdrawal symptoms [20,21,24]. However, we were not able to find studies that have investigated the specific subjective withdrawal symptoms in relation to serum concentrations in MMT, although, such symptoms have been shown in studies on opioid use disorders to be related to opioid abstinence [29-31]. Of the symptoms most often reported in the present study (restlessness, hot- or cold flushes, anxiety, nose run and bone- and muscle ache), only anxiety, bone- and muscle ache, restlessness and shaking were related to serum methadone concentration.

Although the present study is not designed to could confirm possible causalities and the dose-response relationship with serum methadone concentration is not very strong, the findings could imply the need for dose escalation when these specific subjective withdrawal symptoms are reported by the patients. At least, the clinical significance of the findings may be high for the individual patient's treatment by relieving the unpleasant symptoms. Dose escalations may be considered even if the dose already given is high, keeping in mind alternations in methadone pharmacokinetics that may influence the serum concentration reached by each particular patient $[12,14-16]$. However, expected treatment benefits of optimizing methadone dose must always be balanced against the risk of severe harms like death due to overdose and risk of methadone diversion [32].

A lower CDR among more than half of the study participants who also experienced withdrawal symptoms is a remarkable finding, meaning that serum concentrations were lower in this group despite having comparable methadone doses with those who did not report such symptoms. This may in part be explained by the fact that increasing the dose was not met by a corresponding increase in serum methadone concentration. Considering possible aberrant methadone metabolisms or influence of other disturbing factors, an individualized dose optimization based on appropriate risk-benefit assessments might be emphasized to achieve the treatment effects $[5-8,12]$. Among those not experiencing optimal effect despite increasing the dose, dividing the daily dose or switching to another opioid can be considered $[11,24]$. When none of these measures can help, other causes such as pharmacodynamic factors and genetic variations affecting opioid receptors might be excluded [6, 33]. Finally, diversion of prescribed methadone take-home doses may be considered as an explanation for lower serum concentrations despite receiving appropriate doses and frequently observed medications that we have recorded in the present study.

The higher use of heroin among those with lower serum methadone concentrations, also is in line with earlier studies [20,21, 23], indicating that even heroin use may prompt physicians to consider dose escalation. Using higher methadone doses is shown to be more effective in reducing heroin use and improved treatment retention [34-36]. However, some patients continue using heroin for its euphoric effects or for other reasons, regardless of serum methadone concentration. In addition, it is not clear if the lower serum concentration has caused the heroin use, or if the dose intentionally is not escalated to 
allow the heroin to be felt. It is challenging to answer these questions considering the naturalistic design of the study. Our finding of higher alcohol intake in those with lower serum methadone concentrations could be explained by self-experienced replacement to alleviate opioid withdrawal symptoms. An overlapping effect of alcohol on mu-opioid receptors in the central nerves system has also been demonstrated [37]. In addition, a regular low dose alcohol intake ( $<4$ alcoholic drink/day) may induce the P450 enzymes and thus decrease serum methadone concentration [26]. Considering the increased risk of adverse effects and overdose by concurrent use of opioids and alcohol, the importance of a balanced dose optimization to increase treatment retention and avoid relapse but also toxicity, might be furtherly emphasized.

All participants reported at least one subjective adverse effect related to methadone treatment, the most frequently being concentration difficulties, feeling "brain fog", reduced sexual desire, perspiring and nausea, which are known adverse effects of high doses of opioids including methadone [26-28]. None of the specific adverse effects were significantly related to serum methadone concentration, except for a slight association with nausea. Studies on associations between serum methadone concentration and subjective adverse medication effects in MMT are lacking. Nevertheless, is important to keep in mind other possible physical and psychosocial conditions surrounding the patient, which may influence the total subjective experiences and satisfaction with the treatment [10]. The inability to identify such associations in the present study may also be due to investigating on the group level. Following individuals over time with different doses may have revealed the real picture. Also, we used self-reports by locally developed questionnaire on common subjective adverse effects, not by a validated instrument, which could have been better in picking up possible adverse effects.

The participants with higher serum methadone concentrations reported to use more cannabis. A possible explanation may be a central acting effect to counterbalance some undesirable side effects related to methadone treatment, for instance an antiemetic effect by cannabis [38]. Another speculation can be a desire to get high using cannabis among those with higher serum methadone concentrations and accordingly more suppressing effects on the central nervous system. Some researchers have also suggested an opioid saving effect of cannabis in patients with opioid dependence [39]; however, in a review research [40], use of cannabis was not associated with retention in MMT and could not predict treatment outcomes such as relapse to heroin use or psychosocial functioning. To our knowledge, an association with methadone dose or serum concentration and cannabis use currently is not investigated in clinical studies.

\section{Strengths and limitations}

Due to the prospective nature of our study and having the available platform to continuously get access to the patients or repeat the records if needed, we were able to manage the data collection more closely and reduce information bias. This might be considered as a strength of the study. On the other hand, due to naturalistic nature of the study and being aware of the instability in the patient group with a chaotic life for most of them often affected by various emergencies, we were not able to perform all the 
interviews and blood tests at the same time (delayed follow-up) as some of the patients did not meet to the planned interviews or were not able to complete the measurements at the same time. As the result, only approximately half of those who had completed the primary surveys were eligible to be included in the statistical analyses. This may be considered as a limitation of the study, although, it illustrates the real life and challenges related to research on populations with severe substance use disorders and related comorbidities. Furthermore, although some associations have been found between some of the subjective symptoms and serum methadone concentration, the results must be interpreted in the light of a small sample size and possible influences of other factors such as current use or abstinence from illicit drugs, comorbid somatic and psychiatric conditions or even manipulating the symptoms to get higher methadone doses. Further clinical studies are needed to achieve more knowledge in this field.

\section{Conclusions}

The added value of the present study is to help clinicians in understanding the association between selfperceived symptoms and serum methadone concentration in MMT. More subjective opioid withdrawal symptoms, particularly anxiety, bodily pain, restlessness and shaking, also more use of heroin and alcohol, but less cannabis use seemed to be related to lower serum methadone concentrations. These findings suggest that methadone dose adjustment mainly can be based on patient's self-reported symptoms and current clinical condition. Measurement of serum concentration is not a necessity for this reason but can be used as a support to clinical decision based on individual assessments. This may be considered as an important clinical implication of the present study focusing on the subjective rather than objective data. In addition, involving the patients in their care may improve the treatment alliance and contribute to the overall well-being among most of them. Probably, this will increase retention in MMT and reduce the risk of relapse and overdose.

\section{Abbreviations}

CDR: Concentration-to-dose ratio; Cl: Confidence interval; DOT: Direct observed treatment; LMM: Linear mixed model; MMT: Methadone maintenance treatment; OAT: Opioid agonist therapy; SOWS: Subjective opioid withdrawal scale; TDM: Therapeutic drug monitoring.

\section{Declarations}

\section{Acknowledgment}

We would especially like to thank all the research nurses in Intro-HCV, Bergen Addiction Research group and all the clinical staff at Department of Addiction Medicine, Section for Opioid Agonist Treatment, Haukeland University Hospital for their incredible efforts on data collection including all clinical interviews and surveys, blood samplings and close follow-up of the participants. We would also like to thank the laboratory staff at Department of Medical Biochemistry and Clinical Pharmacology, Haukeland 
University Hospital, Bergen for the analyses of methadone samples and for a close cooperation during the project period.

\section{Authors' contributions}

FC, LTF, CO, GH, EML, KAJ and JGB have been involved in design of the study, contributed to implementation and writing protocol. FC, JGB and GH conceived the study and designed it primarily. FC is the principal investigator, led statistical analyses and wrote the first draft for the paper. All authors (FC, LTF, CO, GH, JHV, CFA, EML, KAJ and JGB) participated in interpretation of the data, reviewed the manuscript for intellectual content, and approved the final version of the manuscript.

\section{Funding}

This work was supported by the research funding of Western Health Region, Norway [project number F11328 - 912126]. The funder had no role in the study design, data collection and analysis, decision to publish, or preparation of the manuscript. The authors are funded by their respective affiliations.

\section{Availability of data and materials}

All data generated or analyzed during this study are included in this manuscript. No additional data are available due to data protection requirements.

\section{Ethics approval and consent to participate}

The study was approved by the Regional Committee for Medical and Health Research Ethics in Vest Norway (approval No. 2017/297/REK vest). All participants had signed a written informed consent on agreement about using of routine and research data for this purpose and for taking part in the study.

\section{Consent for publication}

Participants have consented for publication.

\section{Competing interests}

The authors declare that they have no competing interests.

\section{Author details}

${ }^{1}$ Department of Addiction Medicine, Haukeland University Hospital, Bergen, Norway

${ }^{2}$ Department of Global Public Health and Primary Care, University of Bergen, Norway

${ }^{3}$ Department of Forensic Medicine, Oslo University Hospital, Oslo, Norway

${ }^{4}$ Center for psychopharmacology, Diakonhjemmet hospital, Oslo, Norway 
${ }^{5}$ Norwegian Center for Addiction Research, University of Oslo, Norway

${ }^{6}$ Institute of clinical psychology, University of Bergen, Norway

${ }^{7}$ Division of Psychiatry, Haukeland University Hospital, Bergen, Norway

${ }^{8}$ Institute of Clinical Medicine, UiT - Norway's Arctic University, Troms $\varnothing$, Norway

${ }^{9}$ Department of Alcohol, Tobacco and Drugs, Norwegian Institute of Public Health, Oslo, Norway

\section{References}

1. Amato L, Davoli M, Perucci CA, Ferri M, Faggiano F, Mattick RP. An overview of systematic reviews of the effectiveness of opiate maintenance therapies: available evidence to inform clinical practice and research. J Subst Abuse Treat. 2005;28:321-9. Review.

2. Gjersing L, Bretteville-Jensen AL. Is opioid substitution treatment beneficial if injecting behaviour continues? Drug Alcohol Depend. 2013;133:121-6.

3. Ma J, Bao YP, Wang RJ, Su MF, Liu MX, Li JQ, et al. Effects of medication-assisted treatment on mortality among opioids users: a systematic review and meta-analysis. Mol Psychiatry 2019;24:1868-83.

4. Sordo L, Barrio G, Bravo MJ, Indave BI, Degenhardt L, Wiessing L, Ferri M, Pastor-Barriuso R. Mortality risk during and after opioid substitution treatment: systematic review and meta-analysis of cohort studies. BMJ. 2017;6:357. Review.

5. Fareed A,Casarella J, Amar R, Vayalapalli S, Drexler K. Methadone Maintenance Dosing Guideline for Opioid Dependence, a Literature Review. J Addict Dis. 2010;29:1-14.

6. Eap CB, Buclin T, Baumann P. Interindividual variability of the clinical pharmacokinetics of methadone: implications for the treatment of opioid dependence. Clin. Pharmacokinet. 2002;41:1153-93.

7. Lehotay DC, George S, Etter ML, Graybiel K, Eichhorst JC, Fern B, WildenboerW, Selby P, Kapur B. Free and bound enantiomers of methadone and its metabolite, EDDP in methadone maintenance treatment: relationship to dosage? Clin. Biochem. 2005;38:1088-94.

8. Maxwell S, Shinderman MS. Optimizing long-term response to methadone maintenance treatment: a 152-week follow-up using higher-dose methadone. J. Addict. Dis. 2002;21:1-12.

9. Bart G. Maintenance Medication for Opiate Addiction: The Foundation of Recovery. J Addict Dis. 2012;31:207-25.

10. Muller AE, Bjornestad R, Clausen T. Dissatisfaction with opioid maintenance treatment partly explains reported side effects of medications. Drug Alcohol Depend. 2018;187:22-8.

11. Leavitt SB, Shinderman M, Maxwell S, Eap CB, Paris P. When "enough" is not enough: new perspectives on optimal methadone maintenance dose. Mt Sinai J Med. 2000;67:404-11. 
12. Mouly S, Bloch V, Peoc'h K, Houze P,Labat L,Ksouda K,et al. Methadone dose in heroin-dependent patients: role of clinical factors, comedications, genetic polymorphisms and enzyme activity. $\mathrm{Br} \mathrm{J}$ Clin Pharmacol. 2015;79:967-77.

13. Ward J, Hall W, Mattick RP. Role of methadone treatment in opioid dependence. Lancet 1999;353:221-6.

14. Soyka M, Kranzler HR, van den Brink W, Krystal J, Moller HJ, Kasper S. The World Federation of Societies of Biological Psychiatry (WFSBP) guidelines for the biological treatment of substance use and related disorders. Part 2: opioid dependence. World J Biol Psychiatry 2011;12:160-87.

15. Chalabianloo F, Westin AA, Skogvoll E, Bramness JG, Spigset O. Methadone serum concentrations and influencing factors: A naturalistic observational study. Psychopharmacology (Berl) 2019;236:3159-67.

16. Kringen MK, Chalabianloo F, Bernard JP, Bramness JG, Molden E, Høiseth G. Combined effect of CYP2B6 genotype and other candidate genes on a steady-state serum concentration of methadone in opioid maintenance treatment. Ther Drug Monit. 2017;39:550-55.

17. Gagajewski A, Apple FS. Methadone-related deaths in Hennepin County Minnesota: 1992-2002. J Forensic Sci. 2003;48:1-4.

18. Mannaioni G, Lanzi C, Lotti M, Galli V, Totti A, Pacileo I, et al. Methadone Dose Adjustments, Plasma R-Methadone Levels and Therapeutic Outcome of Heroin Users: A Randomized Clinical Trial. Eur Addict Res. 2018;24:9-18.

19. Peng S, Jiang H, Du J, Lin S, Pan S, Yu S, Zhao M. Methadone Dosage and Plasma Levels, SNPs of OPRM1 Gene and Age of First Drug Use Were Associated With Outcomes of Methadone Maintenance Treatment. Front Genet. 2018;9:450.

20. Crettol S, Déglon J-J, Besson J, Croquette-Krokkar M, Gothuey I, Hämmig R, MonnatM, HüttemannH, Baumann P, Eap CB. Methadone enantiomer plasma levels, CYP2B6, CYP2C19, and CYP2C9 genotypes, and response to treatment. Clin. Pharmacol. Ther. 2005;78:593-604.

21. Ries RK, Fiellin DA, Miller SC, Saitz R. Principles of Addiction Medicine. 4th ed. Philadelphia: Medicine \& Health Sciences; 2009.

22. Dole VP. Implications of methadone maintenance for theories of narcotic addiction. JAMA. 1988;260:3025-29.

23. Eap CB, Bourquin M, Martin J, Spagnoli J, LivotiS, PowellK, Baumann P, Déglon J. Plasma concentrations of the enantiomers of methadone and therapeutic response in methadone maintenance treatment. Drug Alcohol Depend. 2000;61:47-54.

24. Dyer KR, Foster DJ, White JM, Somogyi AA, Menelaou A, Bochner F. Steady-state pharmacokinetics and pharmacodynamics in methadone maintenance patients: comparison of those who do and do not experience withdrawal and concentration-effect relationships. Clin Pharmacol Ther. 1999;65:68594.

25. Handelsman L, Cochrane KJ, Aronson MJ, Ness R, Rubinstein KJ, Kanof PD. Two new rating scales for opiate withdrawal. Am J Drug Alcohol Abuse. 1987;13:293-308. 
26. Kreek MJ, Borg L, Ducat E, Ray B. Pharmacotherapy in the treatment of addiction: methadone. J Addict Dis. 2010;29:200-16.

27. Macey TA, Weimer MB, Grimaldi EM, Dobscha SK, Morasco BJ. Patterns of Care and Side Effects for Patients Prescribed Methadone for Treatment of Chronic Pain. J Opioid Manag. 2013;9:325-333.

28. Els C, JacksonTD, Kunyk D, LappiVG, Sonnenberg B, Hagtvedt R, Sharma S, Kolahdooz F, Straube S. Adverse events associated with medium- and long-term use of opioids for chronic non-cancer pain: an overview of Cochrane Reviews. Cochrane Database Syst Rev. 2017;10:Cd012509.

29. Gowing L, Ali R, White J. Buprenorphine for the management of opioid withdrawal. Cochrane Database Syst Rev. 2002;2:Cd002025.

30. Hassanian-Moghaddam H, Afzali S, Pooya A. Withdrawal syndrome caused by naltrexone in opioid abusers. Hum Exp Toxicol. 2014;33:561-7.

31. Koob GF. Neurobiological substrates for the dark side of compulsivity in addiction. Neuropharmacology 2009;56 Suppl 1:18-31.

32. Bernard J-P, Havnesl, Slørdal L, WaalH, Mørland J, Khiabani HZ. Methadone-related deaths in Norway. Forensic Sci Int. 2013;10:111-6.

33. Levran O, Peles E, Randesi M, Shu X, Ott J, Shen P-H, Adelson M, Kreek MJ. Association of genetic variation in pharmacodynamic factors with methadone dose required for effective treatment of opioid addiction. Pharmacogenomics 2013;14.

34. Donny EC, Walsh SL, Bigelow GE, Eissenberg T, Stitzer ML. High-dose methadone produces superior opioid blockade and comparable withdrawal suppression to lower doses in opioid dependent humans. Psychopharmacology (Berl) 2002;161:202-12.

35. Donny EC, Brasser SM, Bigelow GE, Stitzer ML, Walsh SL. Methadone doses of $100 \mathrm{mg}$ or greater are more effective than lower doses at suppressing heroin self-administration in opioid-dependent volunteers. Addiction 2005;100:1496-509.

36. Preston K.L, Umbrichat A, Epstein DH. Methadone dose increase and abstinence reinforcement for treatment of continued heroin use during methadone maintenance. Archives of General Psychiatry 2000;57:395-404.

37. Berrettini W. Alcoholaddiction and the mu-opioidreceptor. Prog Neuropsychopharmacol Biol Psychiatry 2015;65:228-33.

38. Whiting PF, Wolff RF, Deshpande S, Di Nisio M, Duffy S, Hernandez AV. Cannabinoidsfor Medical Use: A Systematic Review and Meta-analysis. JAMA. 2015;313:2456-73.

39. Petry NM, Bickel WK. Polydrug abuse in heroin addicts: a behavioral economic analysis. Addiction 1998;93:321-35.

40. Epstein DH, Preston KL. Does Cannabis Use Predict Poor Outcome for Heroin-Dependent Patients on Maintenance Treatment? A Review of Past Findings, and More Evidence Against. Addiction 2003;98:269-79. 
Figures

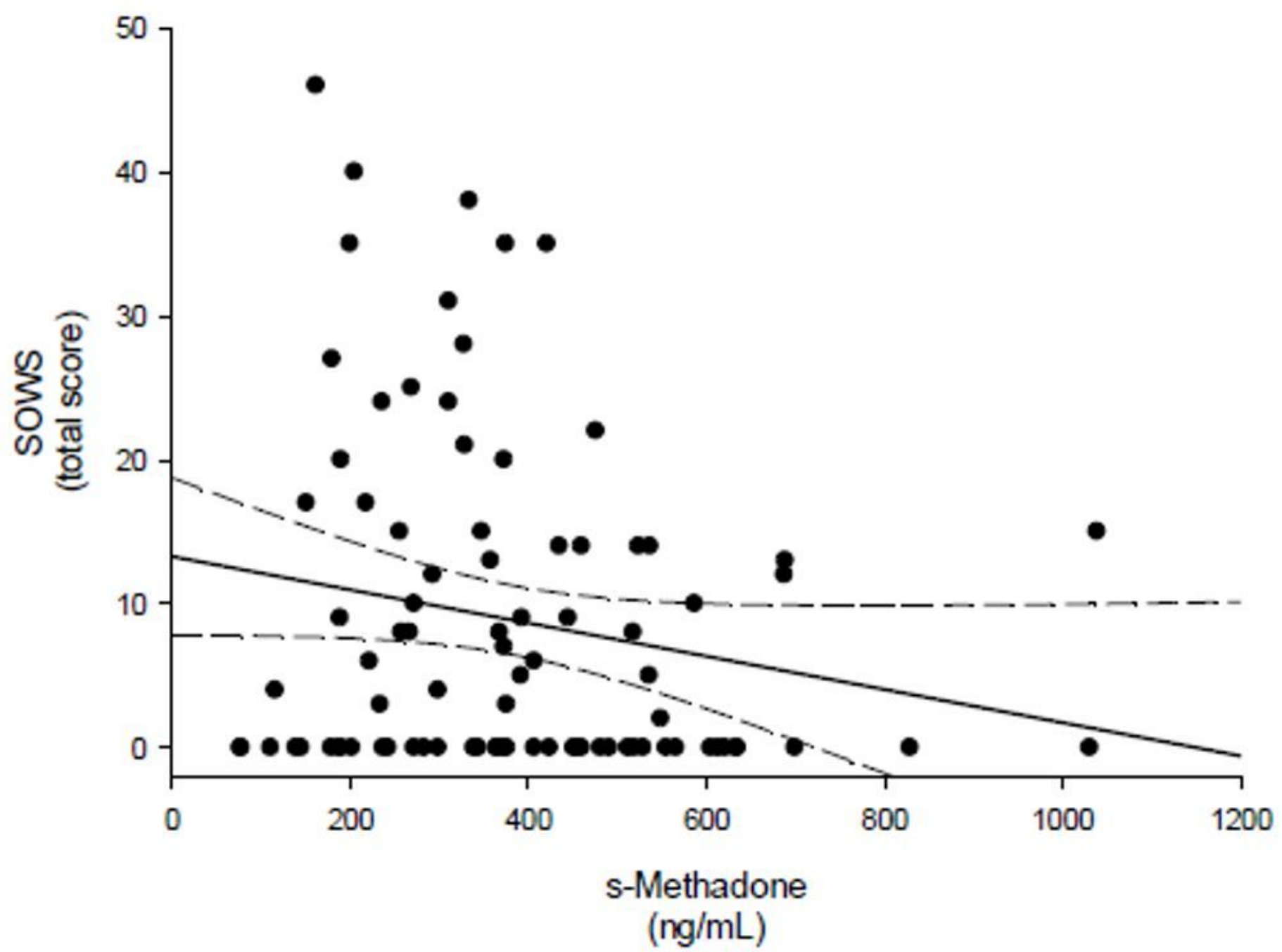

Figure 1

Scatter plot with regression line by recorded total SOWSa scores and measured serum methadone concentrations ( $\mathrm{n}=89$ measurements from 83 participants). The solid and dashed lines represent the regression line and $95 \%$ confidence intervals. a Subjective opioid withdrawal symptoms 